\title{
On the annihilators and attached primes of top local cohomology modules
}

\author{
Ali Atazadeh, Monireh Sedghi, and Reza Naghipour
}

\section{Dedicated to Professor Hossein Zakeri}

\begin{abstract}
Let $\mathfrak{a}$ be an ideal of a commutative Noetherian ring $R$ and $M$ a finitely generated $R$-module. It is shown that $\operatorname{Ann}_{R}\left(H_{\mathfrak{a}}^{\operatorname{dim} M}(M)\right)=$ $\operatorname{Ann}_{R}\left(M / T_{R}(\mathfrak{a}, M)\right)$, where $T_{R}(\mathfrak{a}, M)$ is the largest submodule of $M$ such that $\operatorname{cd}\left(\mathfrak{a}, T_{R}(\mathfrak{a}, M)\right)<\operatorname{cd}(\mathfrak{a}, M)$. Several applications of this result are given. Among other things, it is shown that there exists an ideal $\mathfrak{b}$ of $R$ such that $\operatorname{Ann}_{R}\left(H_{\mathfrak{a}}^{\operatorname{dim} M}(M)\right)=\operatorname{Ann}_{R}\left(M / H_{\mathfrak{b}}^{0}(M)\right)$. Using this, we show that if $H_{\mathfrak{a}}^{\operatorname{dim} R}(R)=0$, then $\operatorname{Att}_{R} H_{\mathfrak{a}}^{\operatorname{dim} R-1}(R)=\{\mathfrak{p} \in$ $\operatorname{Spec} R \mid \operatorname{cd}(\mathfrak{a}, R / \mathfrak{p})=\operatorname{dim} R-1\}$. These generalize the main results of Bahmanpour et al. (see [2, Theorem 2.6]), Hellus (see [7, Theorem 2.3]), and Lynch (see [10, Theorem 2.4]).
\end{abstract}

Mathematics Subject Classification (2010). 13D45, 14B15, 13E05.

Keywords. Annihilator, Attached primes, Cohomological dimension, Local cohomology.

1. Introduction. Let $R$ be an arbitrary commutative Noetherian ring (with identity), $\mathfrak{a}$ an ideal of $R$, and let $M$ be a finitely generated $R$-module. An important problem concerning local cohomology is determining the annihilators of the $i^{\text {th }}$ local cohomology module $H_{\mathfrak{a}}^{i}(M)$. This problem has been studied by several authors; see for example [9-11,14-16], and has led to some interesting results. More recently, in [2] Bahmanpour et al. proved an interesting result about the annihilator $\operatorname{Ann}_{R}\left(H_{\mathfrak{m}}^{d}(M)\right)$ in the case $(R, \mathfrak{m})$ is a complete local ring of dimension $d$.

The purpose of the present paper is to establish some new results concerning the annihilators of local cohomology modules $H_{\mathfrak{a}}^{i}(M)\left(i \in \mathbb{N}_{0}\right)$, where $\mathfrak{a}$ is an ideal in a Noetherian ring $R$ and $M$ a finitely generated module over $R$.

The research of the R. Naghipour was supported in part by a grant from IPM. 
As a main result in the second section, we determine the annihilators of the top local cohomology module $H_{\mathfrak{a}}^{\operatorname{dim}} M(M)$. More precisely, we shall prove the following theorem, which is a generalization of the main result of $[2$, Theorem 2.6] for an arbitrary ideal $\mathfrak{a}$ of an arbitrary Noetherian ring $R$.

Theorem 1.1. Let $R$ be a Noetherian ring, and let $M$ be a finitely generated $R$ module. Then for any ideal $\mathfrak{a}$ of $R, \operatorname{Ann}_{R}\left(H_{\mathfrak{a}}^{\operatorname{dim} M}(M)\right)=\operatorname{Ann}_{R}\left(M / T_{R}(\mathfrak{a}, M)\right)$, where $T_{R}(\mathfrak{a}, M)$ denotes the largest submodule of $M$ such that $\operatorname{cd}\left(\mathfrak{a}, T_{R}(\mathfrak{a}, M)\right)<$ $\operatorname{cd}(\mathfrak{a}, M)$.

The result in Theorem 1.1 is proved in Theorem 2.3. Several corollaries of this result are given. A typical result in this direction is the following, which is a generalization of the main results of $[2$, Theorem 2.6] and [10, Theorem 2.4] for an ideal $\mathfrak{a}$ in an arbitrary Noetherian $\operatorname{ring} R$.

Corollary 1.2. Let $R$ be a Noetherian ring and $\mathfrak{a}$ an ideal of $R$. Let $M$ be a nonzero finitely generated $R$-module of finite dimension c such that $\operatorname{cd}(\mathfrak{a}, M)=c$. Then

$$
\operatorname{Ann}_{R}\left(H_{\mathfrak{a}}^{c}(M)\right)=\operatorname{Ann}_{R}\left(M / H_{\mathfrak{b}}^{0}(M)\right)=\operatorname{Ann}_{R}\left(M / \cap_{\mathrm{cd}\left(\mathfrak{a}, R / \mathfrak{p}_{j}\right)=c} N_{j}\right) .
$$

Here $0=\cap_{j=1}^{n} N_{j}$ denotes a reduced primary decomposition of the zero submodule 0 in $M$ and $N_{j}$ is a $\mathfrak{p}_{j}$-primary submodule of $M$, for all $j=1, \ldots, n$

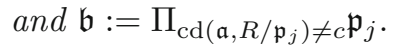

One of the basic problems concerning local cohomology is finding the set of attached primes of $H_{\mathfrak{a}}^{i}(M)$. In Section 3, we obtain some results about the attached primes of local cohomology modules. In this section among other things, we derive the following consequence of Theorem 1.1 and Corollary 1.2, which provides an upper bound for the attached primes of $\operatorname{Att}_{R} H_{\mathfrak{a}}^{\operatorname{cd}(\mathfrak{a}, M)}(M)$. This will generalize the main results of [5] and [4].

Theorem 1.3. Let $R$ be a Noetherian ring and $\mathfrak{a}$ an ideal of $R$. Let $M$ be a finitely generated $R$-module such that $c:=\operatorname{cd}(\mathfrak{a}, M)$ is finite. Then

$$
\operatorname{Att}_{R} H_{\mathfrak{a}}^{c}(M) \subseteq\{\mathfrak{p} \in \operatorname{Supp} M \mid \operatorname{cd}(\mathfrak{a}, R / \mathfrak{p})=c\} .
$$

Moreover, if $c=\operatorname{dim} M$, then

$$
\operatorname{Att}_{R} H_{\mathfrak{a}}^{c}(M)=\left\{\mathfrak{p} \in \operatorname{mAss}_{R} M \mid \operatorname{cd}(\mathfrak{a}, R / \mathfrak{p})=c\right\} .
$$

For an $R$-module $A$, a prime ideal $\mathfrak{p}$ of $R$ is said to be attached prime to $A$ if $\mathfrak{p}=\operatorname{Ann}_{R}(A / B)$ for some submodule $B$ of $A$. We denote the set of attached primes of $A$ by $\operatorname{Att}_{R} A$. This definition agrees with the usual definition of attached prime if $A$ has a secondary representation (cf. [12, Theorem 2.5]).

Another main result in Section 3 is to give a complete characterization of the attached primes of the local cohomology module $H_{\mathfrak{a}}^{\operatorname{dim} R-1}(R)$. More precisely, we shall show the following result, which is an extension as well as a correction of the main theorem of [7].

Theorem 1.4. Let $(R, \mathfrak{m})$ be a local (Noetherian) ring of dimension $d$. Let $\mathfrak{a}$ be an ideal of $R$ such that $\operatorname{dim} R / \mathfrak{a}=1$ and $H_{\mathfrak{a}}^{d}(R)=0$. Then $\operatorname{Assh}_{R} R \subseteq$ 
$\operatorname{Att}_{R} H_{\mathfrak{a}}^{d-1}(R)$. Moreover, if $R$ is complete, then

$\operatorname{Att}_{R} H_{\mathfrak{a}}^{d-1}(R)=\{\mathfrak{p} \in \operatorname{Spec} R \mid \operatorname{dim} R / \mathfrak{p}=d-1$ and $\operatorname{Rad}(\mathfrak{a}+\mathfrak{p})=\mathfrak{m}\} \cup \operatorname{Assh}_{R} R$.

One of our tools for proving Theorem 1.4 is the following:

Proposition 1.5. Let $R$ be a Noetherian ring of finite dimension $d$ and $\mathfrak{a}$ an ideal of $R$ such that $H_{\mathfrak{a}}^{d}(R)=0$. Then

$$
\operatorname{Att}_{R} H_{\mathfrak{a}}^{d-1}(R)=\{\mathfrak{p} \in \operatorname{Spec} R \mid \operatorname{cd}(\mathfrak{a}, R / \mathfrak{p})=d-1\} .
$$

Throughout this paper, $R$ will always be a commutative Noetherian ring with non-zero identity and $\mathfrak{a}$ will be an ideal of $R$. For any $R$-module $M$, the $i^{\text {th }}$ local cohomology module of $M$ with support in $V(\mathfrak{a})$ is defined by

$$
H_{\mathfrak{a}}^{i}(M):=\underset{n \geq 1}{\lim _{n \geq 1}} \operatorname{Ext}_{R}^{i}\left(R / \mathfrak{a}^{n}, M\right) .
$$

The cohomological dimension of $M$ with respect to $\mathfrak{a}$ is defined as

$$
\operatorname{cd}(\mathfrak{a}, M):=\sup \left\{i \in \mathbb{Z} \mid H_{\mathfrak{a}}^{i}(M) \neq 0\right\} .
$$

For each $R$-module $L$, we denote by $\operatorname{Assh}_{R} L\left(\operatorname{resp} \cdot \operatorname{mAss}_{R} L\right)$ the set $\{\mathfrak{p} \in$ $\left.\operatorname{Ass}_{R} L: \operatorname{dim} R / \mathfrak{p}=\operatorname{dim} L\right\}$ (resp. the set of minimal primes of $\operatorname{Ass}_{R} L$ ). Also, we shall use $\operatorname{Att}_{R} L$ to denote the set of attached prime ideals of $L$. For any ideal $\mathfrak{a}$ of $R$, we denote $\{\mathfrak{p} \in \operatorname{Spec} R: \mathfrak{p} \supseteq \mathfrak{a}\}$ by $V(\mathfrak{a})$. Finally, for any ideal $\mathfrak{b}$ of $R$, the radical of $\mathfrak{b}$, denoted by $\operatorname{Rad}(\mathfrak{b})$, is defined to be the set $\left\{x \in R: x^{n} \in \mathfrak{b}\right.$ for some $n \in \mathbb{N}\}$. For any unexplained notation and terminology, we refer the reader to [3] and [13].

2. Annihilators of top local cohomology modules. Let us, firstly, recall the important concept of the cohomological dimension of an $R$-module $L$ with respect to an ideal $\mathfrak{a}$ of a commutative Noetherian $\operatorname{ring} R$, denoted by $\operatorname{cd}(\mathfrak{a}, L)$; it is the largest integer $i$ such that $H_{\mathfrak{a}}^{i}(L) \neq 0$. The main result of this section is Theorem 2.3. The following lemma plays a key role in the proof of that theorem.

Lemma 2.1. Let $R$ be a Noetherian ring and $\mathfrak{a}$ an ideal of $R$. Let $M$ and $N$ be two finitely generated $R$-modules such that $\operatorname{Supp} N \subseteq \operatorname{Supp} M$. Then, $\operatorname{cd}(\mathfrak{a}, N) \leq \operatorname{cd}(\mathfrak{a}, M)$.

Proof. See [6, Theorem 2.2].

Definition 2.2. Let $R$ be a Noetherian ring and $\mathfrak{a}$ an ideal of $R$. Let $M$ be a non-zero finitely generated $R$-module. We denote by $\mathrm{T}_{R}(\mathfrak{a}, M)$ the largest submodule of $M$ such that $\operatorname{cd}\left(\mathfrak{a}, \mathrm{T}_{R}(\mathfrak{a}, M)\right)<\operatorname{cd}(\mathfrak{a}, M)$. It is easy to check that $\mathrm{T}_{R}(\mathfrak{a}, M)=\cup\{N \mid N \leq M$ and $\operatorname{cd}(\mathfrak{a}, N)<\operatorname{cd}(\mathfrak{a}, M)\}$. In particular, for a local ring $(R, \mathfrak{m})$, we denote $\mathrm{T}_{R}(\mathfrak{m}, M)$ by $T_{R}(M)$. Thus $T_{R}(M)=\cup\{N \mid N \leq M$ and $\operatorname{dim} N<\operatorname{dim} M$ \}, see [2, Definition 2.5].

Now, we are prepared to present the main result of this section, which is a generalization of the main result of [2, Theorem 2.6]. 
Theorem 2.3. Let $R$ be a Noetherian ring and $\mathfrak{a}$ an ideal of $R$. Let $M$ be a finitely generated $R$-module with finite dimension $c$ such that $\operatorname{cd}(\mathfrak{a}, M)=c$. Then

$$
\operatorname{Ann}_{R}\left(H_{\mathfrak{a}}^{c}(M)\right)=\operatorname{Ann}_{R}\left(M / T_{R}(\mathfrak{a}, M)\right) .
$$

Proof. It easily follows from the canonical exact sequence

$$
0 \longrightarrow T_{R}(\mathfrak{a}, M) \longrightarrow M \longrightarrow M / T_{R}(\mathfrak{a}, M) \longrightarrow 0
$$

and Lemma 2.1 that $\operatorname{cd}\left(\mathfrak{a}, M / T_{R}(\mathfrak{a}, M)\right)=c$ and

$$
H_{\mathfrak{a}}^{c}(M) \cong H_{\mathfrak{a}}^{c}\left(M / T_{R}(\mathfrak{a}, M)\right)
$$

Thus we have

$$
H_{\mathfrak{a}}^{c}(M) \cong H_{\mathfrak{a}}^{\mathrm{cd}\left(\mathfrak{a}, M / T_{R}(\mathfrak{a}, M)\right)}\left(M / T_{R}(\mathfrak{a}, M)\right) .
$$

Consequently, we can (and do) assume that $T_{R}(\mathfrak{a}, M)=0$, and with this assumption our aim is to show that $\operatorname{Ann}_{R}\left(H_{\mathfrak{a}}^{c}(M)\right)=\operatorname{Ann}_{R}(M)$. To this end, as

$$
\operatorname{Ann}_{R}(M) \subseteq \operatorname{Ann}_{R}\left(H_{\mathfrak{a}}^{c}(M)\right)
$$

it is enough for us to prove that

$$
\operatorname{Ann}_{R / \operatorname{Ann}_{R}(M)}\left(H_{\mathfrak{a}}^{c}(M)\right)=0 .
$$

Since by Lemma $2.1 \operatorname{cd}\left(\mathfrak{a}, R / \operatorname{Ann}_{R}(M)\right)=\operatorname{dim} R / \operatorname{Ann}_{R}(M)=c$, it follows that it is enough for us to show that

$$
\operatorname{Ann}_{R / A_{n}(M)}\left(H_{\mathfrak{a}\left(R / \operatorname{Ann}_{R}(M)\right)}^{c}(M)\right)=0 .
$$

We can, and do, assume henceforth in this proof that $\operatorname{cd}(\mathfrak{a}, R)=c=\operatorname{dim} R$ and that $M$ is a faithful $R$-module. Hence it is sufficient for us to show that $\operatorname{Ann}_{R}\left(H_{\mathfrak{a}}^{c}(M)\right)=0$. To this end, let $x \in \operatorname{Ann}_{R}\left(H_{\mathfrak{a}}^{c}(M)\right)$. Our strategy is to show that $H_{\mathfrak{a}}^{c}(x M)=0$. To do this, it is sufficient for us to show that $H_{\mathfrak{a} R_{\mathfrak{p}}}^{c}\left(x M_{\mathfrak{p}}\right)=0$, for all $\mathfrak{p} \in \operatorname{Spec} R$. If $\operatorname{dim}_{R_{\mathfrak{p}}}\left(x M_{\mathfrak{p}}\right)<c$, then the assertion follows from Grothendieck's vanishing theorem (see [3, Theorem 6.1.2]). Hence we may assume that $\operatorname{dim}_{R_{\mathfrak{p}}}\left(x M_{\mathfrak{p}}\right)=c$. Then $\operatorname{dim}_{R_{\mathfrak{p}}} M_{\mathfrak{p}}=c$. Now, if $H_{\mathfrak{a} R_{\mathfrak{p}}}^{c}\left(M_{\mathfrak{p}}\right)=0$, then it follows from Lemma 2.1 that $H_{\mathfrak{a} R_{\mathfrak{p}}}^{c}\left(x M_{\mathfrak{p}}\right)=0$, and there is nothing to prove. We therefore make the additional assumption that $H_{\mathfrak{a} R_{\mathfrak{p}}}^{c}\left(M_{\mathfrak{p}}\right) \neq 0$. Now, as $x H_{\mathfrak{a} R_{\mathfrak{p}}}^{c}\left(M_{\mathfrak{p}}\right)=0$, it follows from [1, Corollary 3.11] that $H_{\mathfrak{a} R_{\mathfrak{p}}}^{c}\left(x M_{\mathfrak{p}}\right)=0$. Consequently, $H_{\mathfrak{a}}^{c}(x M)=0$. Hence $\operatorname{cd}(\mathfrak{a}, x M)<c$ and since $T_{R}(\mathfrak{a}, M)=0$, we deduce that $x M=0$. Since $M$ is faithful, it follows that $x=0$, as required.

The first application of Theorem 2.3 extends the main result of $[2$, Theorem $2.6]$.

Corollary 2.4. (cf. [2, Theorem 2.6]) Let $(R, \mathfrak{m})$ be a local (Noetherian) ring and $M$ a finitely generated $R$-module. Then $\operatorname{Ann}_{R}\left(H_{\mathfrak{m}}^{\operatorname{dim}} M_{(}(M)\right)=\operatorname{Ann}_{R}\left(M / T_{R}(M)\right)$.

Proof. The result follows from Theorem 2.3 and the standard fact $\operatorname{cd}(\mathfrak{m}, M)=$ $\operatorname{dim} M$. 
Corollary 2.5. Let $R$ be a Noetherian ring with finite dimension $c$ and $\mathfrak{a}$ an ideal of $R$ such that $\operatorname{cd}(\mathfrak{a}, R)=c$. Then

$$
\operatorname{Ann}_{R}\left(H_{\mathfrak{a}}^{c}(R)\right)=T_{R}(\mathfrak{a}, R) .
$$

Proof. The assertion follows from Theorem 2.3.

Remark 2.6. Let $R$ be a Noetherian ring, a an ideal of $R$, and $M$ a nonzero finitely generated $R$-module with finite cohomological dimension $c:=$ $\operatorname{cd}(\mathfrak{a}, M)$. Let $\left\{M_{i}\right\}_{i=0}^{c}$ be a filtration of submodules of $M$ such that for each integer $0 \leq i \leq c, M_{i}$ is the largest submodule of $M$ with $\operatorname{cd}\left(\mathfrak{a}, M_{i}\right) \leq i$. Then $T_{R}(\mathfrak{a}, M)=M_{c-1}$ and by [1, Proposition 2.3], we have

$$
T_{R}(\mathfrak{a}, M)=H_{\mathfrak{b}}^{0}(M)=\cap_{\operatorname{cd}\left(\mathfrak{a}, R / \mathfrak{p}_{j}\right)=c} N_{j},
$$

where $0=\cap_{j=1}^{n} N_{j}$ denotes a reduced primary decomposition of the zero submodule 0 in $M, N_{j}$ is a $\mathfrak{p}_{j}$-primary submodule of $M$, and $\mathfrak{b}=\Pi_{\mathrm{cd}\left(\mathfrak{a}, R / \mathfrak{p}_{j}\right) \neq c} \mathfrak{p}_{j}$.

Corollary 2.7. Let $R$ be a Noetherian ring and $\mathfrak{a}$ an ideal of $R$. Let $M$ be a nonzero finitely generated $R$-module with finite dimension c such that $\operatorname{cd}(\mathfrak{a}, M)=$ c. Then

$$
\operatorname{Ann}_{R}\left(H_{\mathfrak{a}}^{c}(M)\right)=\operatorname{Ann}_{R}\left(M / H_{\mathfrak{b}}^{0}(M)\right)=\operatorname{Ann}_{R}\left(M / \cap_{\operatorname{cd}\left(\mathfrak{a}, R / \mathfrak{p}_{j}\right)=c} N_{j}\right) .
$$

Here $0=\cap_{j=1}^{n} N_{j}$ denotes a reduced primary decomposition of the zero submodule 0 in $M$ and $N_{j}$ is a $\mathfrak{p}_{j}$-primary submodule of $M$ for all $j=1, \ldots, n$ and $\mathfrak{b}=\Pi_{\mathrm{cd}\left(\mathfrak{a}, R / \mathfrak{p}_{j}\right) \neq c} \mathfrak{p}_{j}$.

Proof. The assertion follows from Theorem 2.3 and Remark 2.6.

Corollary 2.8. Let $R$ be a Noetherian ring with finite dimension $c$ and $\mathfrak{a}$ an ideal of $R$ such that $\operatorname{cd}(\mathfrak{a}, R)=c$. Then

$$
\operatorname{Ann}_{R}\left(H_{\mathfrak{a}}^{c}(R)\right)=H_{\mathfrak{b}}^{0}(R)=\cap_{\mathrm{cd}\left(\mathfrak{a}, R / \mathfrak{p}_{j}\right)=c} \mathfrak{q}_{j},
$$

where $0=\cap_{j=1}^{n} \mathfrak{q}_{j}$ is a reduced primary decomposition of the zero ideal of $R$, $\mathfrak{q}_{j}$ is a $\mathfrak{p}_{j}$-primary ideal of $R$ for all $1 \leq j \leq n$, and $\mathfrak{b}=\Pi_{\operatorname{cd}\left(\mathfrak{a}, R / \mathfrak{p}_{j}\right) \neq c} \mathfrak{p}_{j}$.

Proof. The result follows from Corollary 2.7.

Corollary 2.9. Let $R$ be a Noetherian ring with finite dimension $c$ and $\mathfrak{a}$ an ideal of $R$ such that $\operatorname{cd}(\mathfrak{a}, R)=c$. Then the following conditions are equivalent:

(i) $\operatorname{Ann}_{R}\left(H_{\mathfrak{a}}^{c}(R)\right)=0$.

(ii) $\operatorname{Ass}_{R} R=\{\mathfrak{p} \in \operatorname{Spec} R \mid \operatorname{cd}(\mathfrak{a}, R / \mathfrak{p})=c\}$.

Proof. The result follows from Corollary 2.8.

Corollary 2.10. Let $R$ be a Noetherian domain with finite dimension $c$ and $\mathfrak{a}$ an ideal of $R$ such that $\operatorname{cd}(\mathfrak{a}, R)=c$. Then $\operatorname{Ann}_{R}\left(H_{\mathfrak{a}}^{c}(R)\right)=0$.

Proof. Since $\operatorname{Ass}_{R} R=0$, the assertion follows immediately from Corollary 2.9.

Corollary 2.11. Let $(R, \mathfrak{m})$ be a local (Noetherian) ring and $\mathfrak{a}$ an ideal of $R$ such that grade $\mathfrak{a}=\operatorname{cd}(\mathfrak{a}, R)$. Then $\operatorname{Ass}_{R} R \subseteq\{\mathfrak{p} \in \operatorname{Spec} R \mid \operatorname{cd}(\mathfrak{a}, R / \mathfrak{p})=\operatorname{cd}(\mathfrak{a}, R)\}$. 
Proof. Since grade $\mathfrak{a}=\operatorname{cd}(\mathfrak{a}, R)$, it follows from [10, Theorem 3.3] that $\operatorname{Ann}_{R}\left(H_{\mathfrak{a}}^{c}(R)\right)=0$. Moreover, in view of the proof of Theorem 2.3,

$$
H_{\mathfrak{a}}^{c}(R) \cong H_{\mathfrak{a}}^{c}\left(R / T_{R}(\mathfrak{a}, R)\right)
$$

Hence $T_{R}(\mathfrak{a}, R) \subseteq \operatorname{Ann}_{R}\left(H_{\mathfrak{a}}^{c}(R)\right)$, and so $T_{R}(\mathfrak{a}, R)=0$. Now, the assertion follows from Remark 2.6.

Corollary 2.12. Let $R$ be a Noetherian ring and $\mathfrak{a}$ an ideal of $R$. Let $M$ be a finitely generated $R$-module with finite dimension $c$ such that $\operatorname{cd}(\mathfrak{a}, M)=c$ and $x \in R$. Then $H_{\mathfrak{a}}^{c}(x M)=0$ if and only if $x H_{\mathfrak{a}}^{c}(M)=0$. In particular, $\operatorname{Ann}_{R} H_{\mathfrak{a}}^{c}(M)=0$ if and only if $\operatorname{cd}(\mathfrak{a}, r M)=\operatorname{cd}(\mathfrak{a}, M)$ for every non-zero element $r$ of $R$.

Proof. The assertion follows from Theorem 2.3.

Corollary 2.13. Let $R$ be a Noetherian ring and $\mathfrak{a}$ an ideal of $R$. Let $M$ be a finitely generated $R$-module with finite dimension $c$ such that $\operatorname{cd}(\mathfrak{a}, M)=c$. Then

(i) $\operatorname{Ann}_{R}\left(H_{\mathfrak{a}}^{c}(M)\right)=\operatorname{Ann}_{R}(M)$ whenever $\operatorname{Ass}_{R} M \subseteq\{\mathfrak{p} \in \operatorname{Supp} M \mid$ $\operatorname{cd}(\mathfrak{a}, R / \mathfrak{p})=c\}$.

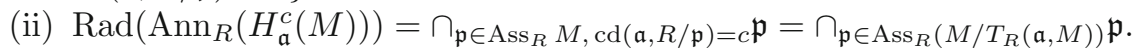

(iii) $V\left(\operatorname{Ann}_{R}\left(H_{\mathfrak{a}}^{c}(M)\right)\right)=\operatorname{Supp}\left(M / T_{R}(\mathfrak{a}, M)\right)$.

Proof. (i) follows from Corollary 2.7. To show (ii), use [1, Proposition 2.6(iii)] and Corollary 2.7. In order to prove (iii), in view of Theorem 2.3 we have

$$
V\left(\operatorname{Ann}_{R} H_{\mathfrak{a}}^{c}(M)\right)=V\left(\operatorname{Ann}_{R} M / T_{R}(\mathfrak{a}, M)\right)=\operatorname{Supp}\left(M / T_{R}(\mathfrak{a}, M)\right) .
$$

3. Attached primes of local cohomology modules. It will be shown in this section that the subjects of the previous section can be used to investigate the attached prime ideals of local cohomology modules. In fact, we will generalize and improve the main result of Hellus (cf. [7, Theorem 2.3]). The main result is Theorem 3.7. The following proposition will serve to shorten the proof of the main theorem. We begin with

Definition 3.1. Let $L$ be an $R$-module. We say that a prime ideal $\mathfrak{p}$ of $R$ is an attached prime of $L$ if there exists a submodule $K$ of $L$ such that $\mathfrak{p}=$ $\operatorname{Ann}_{R}(L / K)$. We denote by $\operatorname{Att}_{R} L$ the set of attached primes of $L$.

When $M$ is representable in the sense of [12] (e.g. Artinian or injective), our definition of $\operatorname{Att}_{R} L$ coincides with that of MacDonald [12], Sharp [17].

It follows easily from the definition that, if $\mathfrak{p} \in \operatorname{Att}_{R} L$, then $L \otimes_{R} R / \mathfrak{p} \neq 0$. This is used in the proof of Theorem 3.3.

Lemma 3.2. Let $R$ be a Noetherian ring and $L$ an $R$-module. Then, the set of minimal elements of $V\left(\operatorname{Ann}_{R}(L)\right)$ coincides with that of $\operatorname{Att}_{R} L$. In particular,

$$
\operatorname{Rad}\left(\operatorname{Ann}_{R}(L)\right)=\cap_{\mathfrak{p} \in \operatorname{Att}_{R} L \mathfrak{p} .}
$$


Proof. The assertion follows from the Definition 3.1 and the fact that $\mathfrak{p}=$ $\operatorname{Ann}_{R}(L / \mathfrak{p} L)$ for each minimal prime $\mathfrak{p}$ over $\operatorname{Ann}_{R}(L)$.

We are now ready to state and prove the first main result of this section, that gives us an upper bound for the attached primes of $\operatorname{Att}_{R} H_{\mathfrak{a}}^{\operatorname{cd}(\mathfrak{a}, M)}(M)$. Before this, we note that as $\operatorname{Ann}_{R}(M) \subseteq \operatorname{Ann}_{R}\left(H_{\mathfrak{a}}^{i}(M)\right)$, it follows that $\operatorname{Att}_{R}\left(H_{\mathfrak{a}}^{i}(M)\right) \subseteq \operatorname{Supp} M$ for every finitely generated $R$-module $M$.

Theorem 3.3. Let $R$ be a Noetherian ring and $\mathfrak{a}$ an ideal of $R$. Let $M$ be a non-zero finitely generated $R$-module such that $c:=\operatorname{cd}(\mathfrak{a}, M)$ is finite. Then

$$
\operatorname{Att}_{R} H_{\mathfrak{a}}^{c}(M) \subseteq\{\mathfrak{p} \in \operatorname{Supp} M \mid \operatorname{cd}(\mathfrak{a}, R / \mathfrak{p})=c\} .
$$

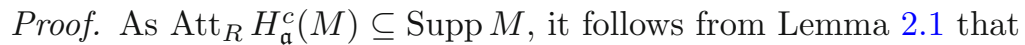

$$
\operatorname{Att}_{R} H_{\mathfrak{a}}^{c}(M) \subseteq\{\mathfrak{p} \in \operatorname{Supp} M \mid \operatorname{cd}(\mathfrak{a}, R / \mathfrak{p}) \leq c\} .
$$

Now, it is enough to show that

$$
\operatorname{Att}_{R} H_{\mathfrak{a}}^{c}(M) \subseteq\{\mathfrak{p} \in \operatorname{Supp} M \mid \operatorname{cd}(\mathfrak{a}, R / \mathfrak{p}) \geq c\} .
$$

To this end, let $\mathfrak{p} \in \operatorname{Att}_{R} H_{\mathfrak{a}}^{c}(M)$. Then

$$
\mathfrak{p} / \operatorname{Ann}_{R}(M) \in \operatorname{Att}_{R / \operatorname{Ann}_{R}(M)} H_{\mathfrak{a}}^{c}(M),
$$

and so

$$
H_{\mathfrak{a}}^{c}(M) \otimes_{R / \operatorname{Ann}_{R}(M)} R / \mathfrak{p} \neq 0 .
$$

Now, as

$$
H_{\mathfrak{a}}^{c}(M) \cong H_{\mathfrak{a}}^{c}\left(R / \operatorname{Ann}_{R}(M)\right) \otimes_{R / \operatorname{Ann}_{R}(M)} M,
$$

it follows that

$$
H_{\mathfrak{a}}^{c}\left(R / \operatorname{Ann}_{R}(M)\right) \otimes_{R / \operatorname{Ann}_{R}(M)} M \otimes_{R / \operatorname{Ann}_{R}(M)} R / \mathfrak{p} \neq 0 .
$$

Consequently

$$
H_{\mathfrak{a}}^{c}(R / \mathfrak{p}) \otimes_{R / \operatorname{Ann}_{R}(M)} M \neq 0,
$$

and thus $H_{\mathfrak{a}}^{c}(R / \mathfrak{p}) \neq 0$, as required.

The next corollary proves again the main result of [5, Theorem 2.5].

Corollary 3.4. Let $R$ be a Noetherian ring and $\mathfrak{a}$ an ideal of $R$. Let $M$ be a non-zero finitely generated $R$-module of finite dimension $d$. Then

$$
\operatorname{Att}_{R} H_{\mathfrak{a}}^{d}(M)=\left\{\mathfrak{p} \in \operatorname{mAss}_{R} M \mid \operatorname{cd}(\mathfrak{a}, R / \mathfrak{p})=d\right\} .
$$

Proof. We can (and do) assume that $H_{\mathfrak{a}}^{d}(M) \neq 0$. Then in view of Theorem 3.3, we have

$$
\operatorname{Att}_{R} H_{\mathfrak{a}}^{d}(M) \subseteq\left\{\mathfrak{p} \in \operatorname{mAss}_{R} M \mid \operatorname{cd}(\mathfrak{a}, R / \mathfrak{p})=d\right\} .
$$

Now, in order to show the inverse containment, let $\mathfrak{p}$ be a prime ideal of $R$ such that $\mathfrak{p} \in \operatorname{mAss}_{R} M$ and $\operatorname{cd}(\mathfrak{a}, R / \mathfrak{p})=d$. Then in view of [1, Proposition 2.6], we have $\mathfrak{p} \in \operatorname{Ass}_{R} M / T_{R}(\mathfrak{a}, M)$, and so by Corollary 2.13(iii), $\mathfrak{p} \in V\left(\operatorname{Ann}_{R}\left(H_{\mathfrak{a}}^{d}(M)\right)\right)$. Hence in view of Lemma 3.2, $\mathfrak{p} \in \operatorname{Att}_{R} H_{\mathfrak{a}}^{d}(M)$, as required. 
Corollary 3.5. Let $R$ be a Noetherian ring and $\mathfrak{a}$ an ideal of $R$. Let $M$ be a finitely generated $R$-module such that $c:=\operatorname{cd}(\mathfrak{a}, R)$ is finite. Then

$$
\begin{aligned}
\{\mathfrak{p} \in \operatorname{Spec} R \mid \operatorname{cd}(\mathfrak{a}, R / \mathfrak{p}) & =c=\operatorname{dim} R / \mathfrak{p}\} \subseteq \operatorname{Att}_{R} H_{\mathfrak{a}}^{c}(R) \\
& \subseteq\{\mathfrak{p} \in \operatorname{Spec} R \mid \operatorname{cd}(\mathfrak{a}, R / \mathfrak{p})=c\} .
\end{aligned}
$$

Moreover, if $c=\operatorname{dim} R$, then $\operatorname{Supp} H_{\mathfrak{a}}^{c}(R) \subseteq V\left(\mathfrak{a}+T_{R}(\mathfrak{a}, R)\right)$.

Proof. In order to prove the first containment, in view of Theorem 3.3, it is enough to show that

$$
\{\mathfrak{p} \in \operatorname{Spec} R \mid \operatorname{cd}(\mathfrak{a}, R / \mathfrak{p})=c=\operatorname{dim} R / \mathfrak{p}\} \subseteq \operatorname{Att}_{R} H_{\mathfrak{a}}^{c}(R) .
$$

To this end, let $\mathfrak{p} \in \operatorname{Spec} R$ be such that $\operatorname{cd}(\mathfrak{a}, R / \mathfrak{p})=c=\operatorname{dim} R / \mathfrak{p}$. Then in view of Corollary 3.4, we have $\mathfrak{p} \in \operatorname{Att}_{R} H_{\mathfrak{a}}^{c}(R / \mathfrak{p})$. Now, from the exact sequence

$$
0 \longrightarrow \mathfrak{p} \longrightarrow R \longrightarrow R / \mathfrak{p} \longrightarrow 0
$$

and the right exactness of $H_{\mathfrak{a}}^{c}(\cdot)$, we deduce that $\mathfrak{p} \in \operatorname{Att}_{R} H_{\mathfrak{a}}^{c}(R)$, as required.

In addition, in order to show the last inclusion, use Corollary 2.5 and the fact that $\operatorname{Supp} H_{\mathfrak{a}}^{c}(R) \subseteq V(\mathfrak{a})$.

Lemma 3.6. Let $R$ be a Noetherian domain of finite dimension $d$ and $\mathfrak{a}$ an ideal of $R$ such that $\operatorname{cd}(\mathfrak{a}, R)=d-1$. Then $\operatorname{Ann}_{R}\left(H_{\mathfrak{a}}^{d-1}(R)\right)=0$.

Proof. Set $J:=\operatorname{Ann}_{R}\left(H_{\mathrm{a}}^{d-1}(R)\right)$. We now suppose that $J \neq 0$ and look for a contadiction. To this end, from the right exactness of the functor $H_{\mathfrak{a}}^{d-1}(\cdot)$, we deduce that $H_{\mathfrak{a}}^{d-1}(R) \cong H_{\mathfrak{a}}^{d-1}\left(R / J^{t}\right)$, for all integers $t$, and so by Lemma 2.1 we have $\operatorname{cd}\left(\mathfrak{a}, R / J^{t}\right)=d-1$. Now, as $R$ is an integral domain and $J^{t} \neq$ 0 , it follows that $\operatorname{dim} R / J^{t}=d-1$ for all integers $t$. Moreover, in view of Corollary 3.4, there is a $\mathfrak{p} \in \operatorname{mAss}_{R} R / J^{t}$ such that $\operatorname{cd}(\mathfrak{a}, R / \mathfrak{p})=d-1$ for all integers $t$. Now, let $\mathfrak{q}_{t}$ be the $\mathfrak{p}$-primary component of $J^{t}$. Then, in view of Corollary 2.7, we have

$$
J=\operatorname{Ann}_{R}\left(H_{\mathfrak{a}}^{d-1}\left(R / J^{t}\right)\right) \subseteq \mathfrak{q}_{t}
$$

for all integers $t$. Consequently, we obtain that

$$
J R_{\mathfrak{p}} \subseteq \bigcap_{t \geq 0} \mathfrak{q}_{t} R_{\mathfrak{p}}=\bigcap_{t \geq 0} J^{t} R_{\mathfrak{p}},
$$

and so the Krull's Intersection Theorem implies that $J R_{\mathfrak{p}}=0$. As $R$ is an integral domain, it follows that $J=0$, which is a contradiction.

Now we are prepared to prove the second main theorem of this section, which is a generalization of the main result of Hellus (cf. [7, Theorem 2.3]).

Theorem 3.7. Let $R$ be a Noetherian ring of finite dimension $d$ and $\mathfrak{a}$ an ideal of $R$ such that $H_{\mathfrak{a}}^{d}(R)=0$. Then

$$
\operatorname{Att}_{R} H_{\mathfrak{a}}^{d-1}(R)=\{\mathfrak{p} \in \operatorname{Spec} R \mid \operatorname{cd}(\mathfrak{a}, R / \mathfrak{p})=d-1\} .
$$

Proof. We can (and do) assume that $H_{\mathfrak{a}}^{d-1}(R) \neq 0$. Then in view of Theorem 3.3 , we have

$$
\operatorname{Att}_{R} H_{\mathfrak{a}}^{d-1}(R) \subseteq\{\mathfrak{p} \in \operatorname{Spec} R \mid \operatorname{cd}(\mathfrak{a}, R / \mathfrak{p})=d-1\} .
$$


Now, let $\mathfrak{p}$ be a prime ideal of $R$ such that $\operatorname{cd}(\mathfrak{a}, R / \mathfrak{p})=d-1$, and so $\operatorname{dim} R / \mathfrak{p} \geq$ $d-1$. Hence, using Corollary 2.10 and Lemma 3.6, we can easily see that $\operatorname{Ann}_{R / \mathfrak{p}} H_{\mathfrak{a}}^{d-1}(R / \mathfrak{p})=0$. Accordingly, we have $\operatorname{Ann}_{R} H_{\mathfrak{a}}^{d-1}(R / \mathfrak{p})=\mathfrak{p}$. Thus, in view of the definition, we have $\mathfrak{p} \in \operatorname{Att}_{R} H_{\mathfrak{a}}^{d-1}(R / \mathfrak{p})$. Therefore, from the exact sequence

$$
0 \longrightarrow \mathfrak{p} \longrightarrow R \longrightarrow R / \mathfrak{p} \longrightarrow 0
$$

and the right exactness of the functor $H_{\mathfrak{a}}^{d-1}(\cdot)$, we deduce that $\mathfrak{p} \in \operatorname{Att}_{R}$ $H_{\mathfrak{a}}^{d-1}(R)$, as required.

The following lemma, which is a consequence of the LichtenbaumHartshorne vanishing theorem, is assistant in the proof of Theorem 3.9.

Lemma 3.8. Let $(R, \mathfrak{m})$ be a complete local (Noetherian) ring and $\mathfrak{a}$ an ideal of $R$. Let $\mathfrak{p}$ be a prime ideal of $R$. Then $\operatorname{cd}(\mathfrak{a}, R / \mathfrak{p})=\operatorname{dim} R / \mathfrak{p}$ if and only if $\operatorname{Rad}(\mathfrak{a}+\mathfrak{p})=\mathfrak{m}$.

Proof. Let $\operatorname{cd}(\mathfrak{a}, R / \mathfrak{p})=\operatorname{dim} R / \mathfrak{p}:=c$. Then $H_{\mathfrak{a}}^{c}(R / \mathfrak{p}) \neq 0$, and so according to the Lichtenbaum-Hartshorne vanishing theorem (see [3, Theorem 8.2.1]), $\operatorname{Rad}(\mathfrak{a}+\mathfrak{p})=\mathfrak{m}$. In order to prove the opposite direction, use [3, Theorems 4.2.1 and 6.1.4].

The next theorem, which is a consequence of Theorem 3.7, improves the main result of $[7$, Theorem 2.3]. If $(R, \mathfrak{m})$ is a local ring, then we use $\hat{R}$ to denote the completion of $R$ with respect to the m-adic topology.

Theorem 3.9. Let $(R, \mathfrak{m})$ be a local (Noetherian) ring of dimension $d$. Let $\mathfrak{a}$ be an ideal of $R$ such that $\operatorname{dim} R / \mathfrak{a}=1$ and $H_{\mathfrak{a}}^{d}(R)=0$. Then

$$
\operatorname{Assh}_{R} R \subseteq \operatorname{Att}_{R} H_{\mathfrak{a}}^{d-1}(R)
$$

If, in addition, $R$ is complete, then

$\operatorname{Att}_{R} H_{\mathfrak{a}}^{d-1}(R)=\{\mathfrak{p} \in \operatorname{Spec} R \mid \operatorname{dim} R / \mathfrak{p}=d-1 \quad$ and $\quad \operatorname{Rad}(\mathfrak{a}+\mathfrak{p})=\mathfrak{m}\} \cup \operatorname{Assh}_{R} R$.

Proof. For the proof of $\operatorname{Assh}_{R} R \subseteq \operatorname{Att}_{R} H_{\mathfrak{a}}^{d-1}(R)$, let $\mathfrak{p} \in \operatorname{Assh}_{R} R$. Then, in view of Theorem 3.7, it is enough to show that $\operatorname{cd}(\mathfrak{a}, R / \mathfrak{p})=d-1$. To do this, as $\operatorname{Assh}_{R}(R)=\left\{\mathfrak{q} \cap R \mid \mathfrak{q} \in \operatorname{Assh}_{\hat{R}}(\hat{R})\right\}, \operatorname{dim} \hat{R} / \mathfrak{a} \hat{R}=1$ and $\operatorname{cd}(\mathfrak{a} \hat{R}, \hat{R} / \mathfrak{p} \hat{R})=$ $\operatorname{cd}(\mathfrak{a}, R / \mathfrak{p})$, by using Lemma 2.1 without loss of generality, we may assume that $R$ is complete. Now, since $H_{\mathfrak{a}}^{d}(R)=0$, it follows that $H_{\mathfrak{a}}^{d}(R / \mathfrak{p})=0$. Thus $\mathfrak{a}+\mathfrak{p}$ is not $\mathfrak{m}$-primary, and $\operatorname{so} \operatorname{dim} R /(\mathfrak{a}+\mathfrak{p})=1($ note that $\operatorname{dim} R / \mathfrak{a}=1)$. Let $\mathfrak{q}$ be a minimal prime over $\mathfrak{a}+\mathfrak{p} \operatorname{such}$ that $\operatorname{dim} R / \mathfrak{q}=1$. Then it is easy to see that $\mathfrak{q}$ is also minimal over $\mathfrak{a}$. Next, as $R$ is catenary, it yields that $\operatorname{dim} R_{\mathfrak{q}} / \mathfrak{p} R_{\mathfrak{q}}=d-1$, and so we have

$$
\left(H_{\mathfrak{a}}^{d-1}(R / \mathfrak{p})\right)_{\mathfrak{q}} \cong H_{\mathfrak{q} R_{\mathfrak{q}}}^{d-1}\left(R_{\mathfrak{q}} / \mathfrak{p} R_{\mathfrak{q}}\right) \neq 0 .
$$

Therefore $H_{\mathfrak{a}}^{d-1}(R / \mathfrak{p}) \neq 0$, and hence $\operatorname{cd}(\mathfrak{a}, R / \mathfrak{p})=d-1$; so that $\operatorname{Assh}_{R} R \subseteq$ $\operatorname{Att}_{R} H_{\mathfrak{a}}^{d-1}(R)$. Consequently, Theorem 3.7 enables us to deduce that $\operatorname{Att}_{R} H_{\mathfrak{a}}^{d-1}(R)$ equals the set

$\{\mathfrak{p} \in \operatorname{Spec} R \mid \operatorname{cd}(\mathfrak{a}, R / \mathfrak{p})=\operatorname{dim} R / \mathfrak{p}=d-1\} \cup\left\{\mathfrak{p} \in \operatorname{Assh}_{R} R \mid \operatorname{cd}(\mathfrak{a}, R / \mathfrak{p})=d-1\right\}$. 
Now, using Lemma 3.8, we see that $\operatorname{Att}_{R} H_{\mathfrak{a}}^{d-1}(R)$ is equal with the set

$$
\begin{aligned}
\{\mathfrak{p} \in \operatorname{Spec} R \mid \operatorname{dim} R / \mathfrak{p} & =d-1 \quad \text { and } \operatorname{Rad}(\mathfrak{a}+\mathfrak{p})=\mathfrak{m}\} \\
\cup\left\{\mathfrak{p} \in \operatorname{Assh}_{R} R \mid \operatorname{cd}(\mathfrak{a}, R / \mathfrak{p})\right. & =d-1\} .
\end{aligned}
$$

Therefore, it follows from Theorem 3.7 and $\operatorname{Assh}_{R} R \subseteq \operatorname{Att}_{R} H_{\mathfrak{a}}^{d-1}(R)$ that $\operatorname{Att}_{R} H_{\mathfrak{a}}^{d-1}(R)=\{\mathfrak{p} \in \operatorname{Spec} R \mid \operatorname{dim} R / \mathfrak{p}=d-1 \quad$ and $\quad \operatorname{Rad}(\mathfrak{a}+\mathfrak{p})=\mathfrak{m}\} \cup \operatorname{Assh}_{R} R$, as required.

Remark 3.10. As a main result, it has been proved in [7, Theorem 2.3], if $(R, \mathfrak{m})$ is a complete local ring of dimension $d$ and $\mathfrak{a}$ an ideal of $R$ such that $\operatorname{dim} R / \mathfrak{a}=1$ and $H_{\mathfrak{a}}^{d}(R)=0$, then

$\operatorname{Att}_{R} H_{\mathfrak{a}}^{d-1}(R)=\{\mathfrak{p} \in \operatorname{Spec} R \mid \operatorname{dim} R / \mathfrak{p}=d-1$ and $\operatorname{Rad}(\mathfrak{a}+\mathfrak{p})=\mathfrak{m}\} \cup \operatorname{Assh}_{R} R$.

The proof of [7, Theorem 2.3] relies heavily on [8, Theorems 2.4 and 2.5], and these results are not true in the case that $d=1$. Indeed, if $(R, \mathfrak{m})$ is a complete local domain of dimension $d=1$, then it is easy to see that these results are not true. Thus [7, Theorem 2.3] needs correction, nevertheless their proofs are valid in the case that $d \geq 2$. However, Theorem 3.9 recovers the corrected version of [7, Theorem 2.3].

We end the paper with the following question:

Question. (i) We have shown in Theorem 3.3 that for an ideal $\mathfrak{a}$ of a Noetherian ring $R$ and a finitely generated $R$-module $M$ of finite cohomological dimension $c$,

$$
\operatorname{Att}_{R} H_{\mathfrak{a}}^{c}(M) \subseteq\{\mathfrak{p} \in \operatorname{Supp} M \mid \operatorname{cd}(\mathfrak{a}, R / \mathfrak{p})=c\} .
$$

Is the above containment an equality?

(ii) In Theorem 2.3 we have determined $\operatorname{Ann}_{R}\left(H_{\mathfrak{a}}^{\operatorname{dim} M}(M)\right)$ ?. ? Is it possible to determine ?? $\operatorname{Ann}_{R}\left(H_{\mathfrak{a}}^{\mathrm{cd}(\mathfrak{a} \text { ?,?M) }}(M)\right)$ ?

Acknowledgements. The authors are deeply grateful to the referee for his or her valuable suggestions on the paper and for drawing the authors' attention to Theorem 2.3 and Remark 2.6. Also, we would like to thank Prof. Kamal Bahmanpour for useful discussions, and also the Institute for Research in Fundamental Sciences (IPM) for the financial support.

Open Access. This article is distributed under the terms of the Creative Commons Attribution License which permits any use, distribution, and reproduction in any medium, provided the original author(s) and the source are credited.

\section{References}

[1] A. Atazadeh, M. Sedghi, and R. Naghipour, Cohomological dimension filtration and annihilators of top local cohomology, preprint.

[2] K. Bahmanpour, J. A'zami, and G. Ghasemi, On the annihilators of local cohomology modules, J. Algebra, 363 (2012), 8-13. 
[3] M.P. Brodmann And R.Y. Sharp, Local cohomology; an algebraic introduction with geometric applications, Cambridge University Press, Cambridge, 1998.

[4] M.T. Dibaei And S. Yassemi, Attached primes of the top local cohomology modules with respect to an ideal, Arch. Math. 84 (2005), 292-297.

[5] K. DivaAni-Aazar, Vanishing of the top local cohomology modules over Noetherian rings, Proc. Indian Acad. Sci. (Math. Sci.) 119 (2009), 23-35.

[6] K. Divanani-Aazar, R. Naghipour, and M. Tousi, Cohomological dimension of certain algebraic varieties, Proc. Amer. Math. Soc. 130 (2002), 3537-3544.

[7] C. Hellus, Attached primes of Matlis duals of local cohomology modules, Arch. Math. 89 (2007), 202-210.

[8] C. Hellus and J. Stückrad, Matlis duals of top local cohomology modules, Proc. Amer. Math. 136 (2008), 489-498.

[9] C. Huneke and J. KoH, Cofiniteness and vanishing of local cohomology modules, Math. Proc. Cambridge Philos. Soc. 110 (1991), 421-429.

[10] L.R. LynCH, Annihilators of top local cohomology, Comm. Algebra, 40 (2012), $542-551$.

[11] G. Lyubeznik, Finiteness properties of local cohomology modules, (an application of D-modules to commutative algebra), Invent. Math. 113 (1993), 41-55.

[12] I.G. MaCDonald, Secondary representations of modules over a commutative rings, Symp. Math. vol. XI (1973), 23-43.

[13] H. Matsumura, Commutative ring theory, Cambridge University Press, Cambridge, UK, 1986.

[14] L.T. NHAN AND T.N. An, On the unmixedness and universal catenaricity of local rings and local cohomology modules, J. Algebra, 321 (2009), 303-311.

[15] L.T. Nhan And T.D.M. Chau, On the top local cohomology modules, J. Algebra, 349 (2012), 342-352.

[16] P. Schenzel, Cohomological annihilators, Math. Proc. Cambridge Philos. Soc. 91 (1982), 345-350.

[17] R.Y. Sharp, Secondary representations for injective modules over commutative Noetherian rings, Proc. Edinburgh Math. Soc. 20 (1976), 143-151.

\section{Ali Atazadeh and Monireh Sedghi}

Department of Mathematics,

Azarbaijan Shahid Madani University,

Tabriz, Iran

e-mail: aalzp2002@yahoo.com

\section{MoniReh Sedghi}

e-mail: m_sedghi@tabrizu.ac.ir;

sedghi@azaruniv.ac.ir 
Reza Naghipour

Department of Mathematics,

University of Tabriz,

Tabriz, Iran

e-mail: naghipour@tabrizu.ac.ir

Reza NAGHipour

School of Mathematics,

Institute for Research in Fundamental Sciences (IPM),

P.O. Box 19395-5746,

Tehran, Iran

e-mail: naghipour@ipm.ir

Received: 21 October 2013 